\title{
Article \\ Effects of Acid-Anhydride-Modified Cellulose Nanofiber on Poly(Lactic Acid) Composite Films
}

\author{
Naharullah Jamaluddin (1), Yu-I Hsu*(D), Taka-Aki Asoh and Hiroshi Uyama*(1) \\ Department of Applied Chemistry, Graduate School of Engineering, Osaka University, 2-1 Yamadaoka, Suita, \\ Osaka 565-0871, Japan; naharullah@chem.eng.osaka-u.ac.jp (N.J.); asoh@chem.eng.osaka-u.ac.jp (T.-A.A.) \\ * Correspondence: yuihsu@chem.eng.osaka-u.ac.jp (Y.-I.H.); uyama@chem.eng.osaka-u.ac.jp (H.U.); Tel.: \\ +81-6-6879-7364 (Y.-I.H. \& H.U.); Fax: +81-6-6879-7367 (Y.-I.H. \& H.U.)
}

check for updates

Citation: Jamaluddin, N.; Hsu, Y.-I;

Asoh, T.-A.; Uyama, H. Effects of

Acid-Anhydride-Modified Cellulose Nanofiber on Poly(Lactic Acid) Composite Films. Nanomaterials 2021, 11, 753. https://doi.org/10.3390/ nano11030753

Academic Editor: Byoung-Suhk Kim

Received: 25 February 2021

Accepted: 15 March 2021

Published: 17 March 2021

Publisher's Note: MDPI stays neutral with regard to jurisdictional claims in published maps and institutional affiliations.

\begin{abstract}
In this study, we investigated the effect of the addition of cellulose nanofiber (CNF) fillers on the performance of poly(lactic acid) (PLA). Modification of the hydroxyl group of cellulose to the acyl group by acid anhydrides changed the compatibility of the CNF with PLA. CNF was modified by acetic anhydride, propionic anhydride, and butyric anhydride to form surface-modified acetylated $\mathrm{CNF}(\mathrm{CNFa})$, propionylated CNF (CNFp), and butyrylated CNF (CNFb), respectively, to improve the compatibility with the PLA matrix. The effects of the different acid anhydrides were compared based on their rates of reaction in the acylation process. PLA with modified cellulose nanofiber fillers formed smoother surfaces with better transparency, mechanical, and wettability properties compared with the PLA/CNF composite film. The effects of CNFa, CNFp, and CNFb on the PLA matrix were compared, and it was found that CNFp was the best filler for PLA.
\end{abstract}

Keywords: cellulose; nanofiber; acid anhydrides; poly(lactic acid); nanocomposite; optical properties; mechanical properties

\section{Introduction}

Bio-based polymers are attracting attention as an alternative to existing materials such as synthetic plastics and petroleum-based materials. The development of bio-based polymers is the main aspect of maintaining a sustainable society because of their renewability and biocompatibility, which is parallel with one of the 17 sustainable development goals. Some plastics can be decomposed by combustion, such as polyhydroxyalkanoate, which is widely applied in tissue engineering applications, and poly(lactic acid) (PLA), which is produced by fermentation of corn or sugarcane [1,2]. PLA has interesting features, such as good processability and transparency [3]. In industry, PLA is currently commercialized as single-use disposal packaging [4]. However, PLA also has several drawbacks that limit its applications, such as low thermal, mechanical, and barrier properties [5]. Therefore, a lot of research has been carried out to enhance and counter these drawbacks by preparing PLA composites [6]. The combination of PLA and cellulose as a nanocomposite is expected to exhibit improved properties by combination of the plastic matrix and cellulose filler. Several types of research have been performed to produce PLA/cellulose composites using different processing methods. In 2008, Alemdar and Sain [7] investigated the structure and thermal properties of PLA/cellulose-whisker nanocomposites by solution casting. In 2007, Iwamoto et al. [8] fabricated a PLA/cellulose-nanofiber (CNF) composite by the extrusion mixing method. Modification of cellulose before composite preparation with PLA has also been widely investigated, and most of the modifications improved dispersion of cellulose in the PLA matrix [9].

In each D-glucose unit of cellulose, there are three active hydroxyl groups. These groups can easily form hydrogen bonds, resulting in cellulose showing hydrophilicity. Cellulose is insoluble in common organic solvents and hydrophobic polymers [10]. After 
modification of the hydroxyl groups of cellulose, the hydrogen bonds are weakened, and cellulose becomes more dispersible in organic solvents and most hydrophobic polymers [11]. In addition, cellulose is one of the best filler materials for polymer composites because of its high mechanical strength and biodegradability, and several functionalizations of cellulose have been studied and developed [12]. Fujisawa et al. [13] reported ionic exchange of (2,2,6,6-tetramethylpiperidin-1-yl)oxyl-oxidized CNF by grafting it with poly(ethylene glycol) using organic solvents such as chloroform, toluene, and tetrahydrofuran. Other modifications of cellulose include esterification of bacterial cellulose (BC), alkylation of micro- and nanocellulose, silanization by 3-methacrysloxypropyltrimethoxysilane, and glyoxalization of BC networks [14-17]. Various modifications of cellulose have been performed to improve the compatibility of cellulose with the polymer matrix $[18,19]$. Recently, our group successfully modified cellulose with citric acid to improve its compatibility with poly(propylene) and PLA resins $[20,21]$. Furthermore, esterification of cellulose to produce hydrophobic products has been widely studied since the early 1980s [22].

The dependence of the esterification effects of CNF on the starting reactants and their degrees of substitutions (DSs) is also an interesting topic. For example, Vice-Garcia et al. [23] investigated the dependence of the main transition of cellulose fatty esters on the length of their aliphatic substituents. In 2009, Crépy et al. [24] investigated the effect of saturated and unsaturated C12 to C18 chains on cellulose fatty esters and prepared polymer films from modified cellulose. Recently, the same group studied a series of fatty acid cellulose esters (FACEs) with various DS values and side-chain lengths from C10 to C16, and they compared the mechanical and chemical properties of each FACE [25]. A review of the various material functionalities based on thermoplastic cellulose and related structural polysaccharide derivatives has been published [26]. In the review, they discussed the approaches for enabling effective thermoplasticization and incorporation of material functionalities, such as single-substituent derivatization, derivatization with multiple substituents, blending of simple derivatives, and graft copolymerization. The influences of short alkyl-chain substituents have also been investigated. For example, $\mathrm{Yu}$ et al. [27] modified cellulose with acid anhydrides to form modified cellulose with C1 to $\mathrm{C} 3$ alkyl-chain substituents, and then investigated their effects on the structure and thermal properties of cellulose-g-polyoxyethylene (2) hexadecyl ether. However, very limited research on comparison of the effects of different alkyl-chain substituents of CNF on PLA composite films has been performed.

In this work, CNF was modified with acetic anhydride (AA), propionic anhydride (PA), and butyric anhydride (BA) to form surface-modified acetylated CNF (CNFa), propionylated $\mathrm{CNF}(\mathrm{CNFp})$, and butyrylated $\mathrm{CNF}(\mathrm{CNFb})$, respectively. The acylation method of the CNF was developed based on our previous research [11]. Fillers of modified CNFs ( $\mathrm{m}-\mathrm{CNFs}$ ) with different DS values and lengths of the alkyl-chain substituents were used to fabricate PLA/m-CNF composite films with various transparency, strength, and wettability values. The aims of this work are to investigate the effect of the acid anhydride on the rate of CNF acylation and the influence of the acylated CNF on the PLA/m-CNF composite film. The acylation reaction time was varied to obtain $\mathrm{m}-\mathrm{CNFs}$ with various DS values and predict the rate of acylation. $\mathrm{CNFa}, \mathrm{CNFp}$, and $\mathrm{CNFb}$ differ in the length of the alkyl-chain substituents of the carbonyl groups (Figure 1), which varies from C1 to C3. The PLA/m$\mathrm{CNF}$ composites were expected to improve the mechanical and wettability properties. We focused on the dispersibility and optical, physical, and hydrophobic properties of the $\mathrm{PLA} / \mathrm{m}$-CNF composite films measured by ultraviolet-visible (UV-vis) spectroscopy, a haze meter, scanning electron microscopy (SEM), tensile strength tests, and water contact angle (WCA) measurements. 
(a) CNF (hydroxyl group)

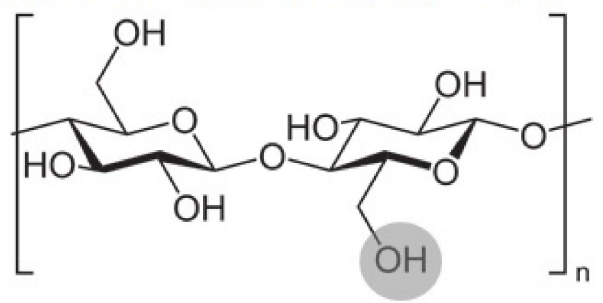

(c) CNFp (propionyl group)

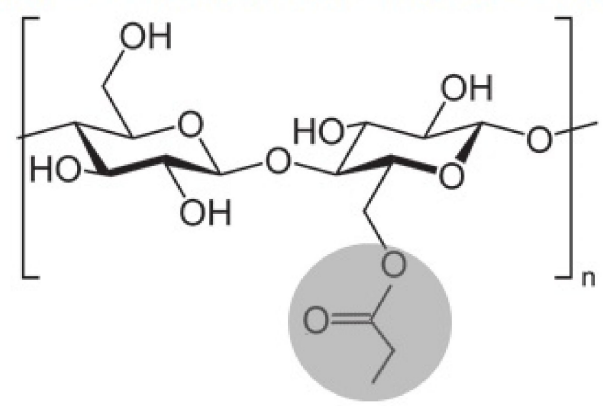

(b) CNFa (acetyl group)

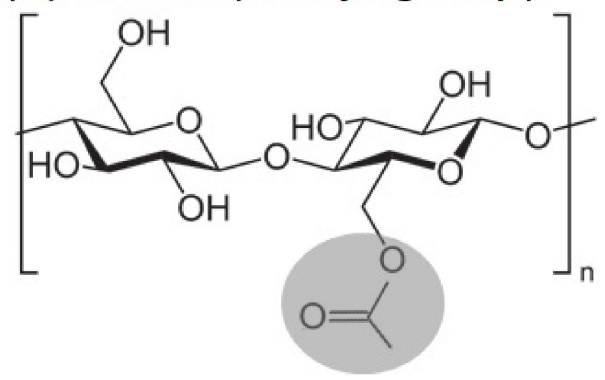

(d) CNFb (butyryl group)

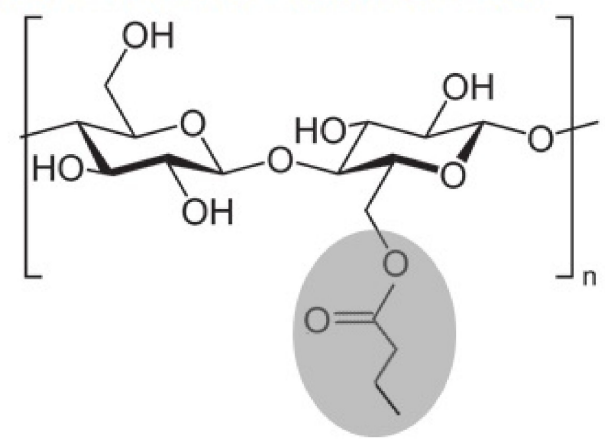

Figure 1. Chemical structures of (a) cellulose nanofiber (CNF); (b) surface-modified acetylated CNF (CNFa); (c) propionylated $\mathrm{CNF}(\mathrm{CNFp})$; and (d) butyrylated $\mathrm{CNF}(\mathrm{CNFb})$.

\section{Materials and Methods}

\subsection{Materials}

Microcrystalline cellulose (MCC) was obtained from Merck Japan Ltd. (Tokyo, Japan). $N, N$-dimethylformamide (DMF) was purchased from Kanto Chemical (Tokyo, Japan). AA, PA, BA, chloroform (>99.0\%), and acetone (>99.0\%) were obtained from Wako Pure Chemical Industries (Osaka, Japan) and used without further treatment. PLA (PLA2003D Ingeo biopolymer) was purchased from NatureWorks LLC (Minnesota, MN, USA). Water was treated with a Model III instrument from Organo Corporation (Tokyo, Japan) to produce deionized water $\left(\mathrm{H}_{2} \mathrm{O}\right)$.

\subsection{Preparation of $\mathrm{CNF}$}

Fibrillation of MCC was performed with a stone grinding machine (Masuko Sangyo, Saitama, Japan) without other chemical treatments. In brief, MCC (40 g) was soaked and stirred in water for 2 days to produce a suspension of $2 \mathrm{wt} \% \mathrm{MCC}$. The MCC suspension was then ground at $1500 \mathrm{rpm}$ for 10 cycles with a stone grinder grit size of 80 (ultra-fine). As the fiber size decreased, MCC automatically exited the chute in the form of CNF. Each cycle was repeated when the hopper was almost empty by transferring the product of the previous cycle back into the hopper. After 10 cycles, the suspension of CNF $(1.57 \mathrm{wt} \%)$ in water was stored in a glass bottle and kept in a refrigerator $\left(<4{ }^{\circ} \mathrm{C}\right)$.

\subsection{Surface Modification of CNF}

First, $0.81 \mathrm{~g}$ of CNF in water (1.57 wt $\%$ ) was homogenized with $80 \mathrm{~mL}$ of DMF. The CNF suspension in this mixed solvent was transferred to a rotary evaporator to remove the water from the system. The mixture was then transferred into a round-bottomed flask connected to a reflux condenser on a hotplate heated to $110{ }^{\circ} \mathrm{C}$ using silicon oil, and simultaneously $0.5 \mathrm{~mol}$ of AA, PA, or BA was added. The acylation reaction time varied from 1 to $4 \mathrm{~h}$ to investigate the reaction rate. The solution was then quenched in an 
ice bath followed by addition of acetone $(50 \mathrm{~mL})$. The solution was then centrifuged and washed several times with acetone to remove the unreacted chemicals and DMF. Finally, the medium was exchanged with chloroform to obtain the $\mathrm{m}-\mathrm{CNF}$ in chloroform. The products were stored in a refrigerator $\left(<4^{\circ} \mathrm{C}\right)$ before preparation of the PLA $/ \mathrm{m}-\mathrm{CNF}$ composite films. The products of the m-CNFs are called CNFaX (AA modified), CNFpX (PA modified), and $\mathrm{CNFbX}$ (BA modified) based on their anhydride, where $X$ is the reaction time (in $\mathrm{h}$ ).

\subsection{Preparation of PLA/m-CNF Composites}

The solution casting method was used to prepare the PLA/m-CNF composites. First, a dispersion of unmodified CNF or $\mathrm{m}-\mathrm{CNF}$ in chloroform ( $0.02 \mathrm{~g}$ of solid) was poured into a beaker, and chloroform was added to reach $50 \mathrm{~g}$ in total weight. Next, $2 \mathrm{~g}$ of PLA was added, and the mixture was stirred for $3 \mathrm{~h}$ at room temperature. The mixture was then poured into a Petri dish and left overnight in a closed bio-shaker at $25 \mathrm{rpm}$ and $40{ }^{\circ} \mathrm{C}$ to evaporate the chloroform. The samples are denoted neat PLA (without filler), PLA/CNF, $\mathrm{PLA} / \mathrm{CNFa}$, PLA/CNFp, and PLA/CNFb composite films.

\subsection{Characterization}

An attenuated total reflection infrared (ATR-IR) spectrometer (iD5 ATR, Thermo Scientific, Waltham, MA, USA) was used to determine the functional groups after modification. Electron-dispersive X-ray (EDX) spectroscopy was carried out to calculate the DS values of the produced m-CNFs using a Miniscope TM3000/SwiftED3000 system (Hitachi, Tokyo, Japan). For the DS calculation, $\mathrm{C}$ and $\mathrm{O}$, but not $\mathrm{H}$, were included. After acylation, the mass ratio of $C$ is expected to increase relative to $O$ owing to the increased $C$ species from the acyl groups that substitute for the $\mathrm{H}$ atoms of the hydroxyl groups of CNF. The transmittance and transparency values of the composite films were determined using a UV-vis spectrophotometer (U-2810, Hitachi, Tokyo, Japan) in the visible region (200-800 nm) scanned at a rate of $800 \mathrm{~nm} / \mathrm{min}$. The transparency values were calculated by

$$
\text { Transparency }=\log \frac{T \text { (average) }}{X}
$$

where $T$ (average) and $X$ are the average UV-vis transmittance and average thickness of the film, respectively. A haze meter (NDH 4000, Nippon Denshoku) was used to calculate the haze values of the PLA/m-CNF composite films. The haze value was determined by

$$
\text { Haze }=\frac{\text { T.T. }- \text { P.T. }}{\text { T.T. }} \times 100
$$

where T.T. and P.T. are the intensities of the transmitted light and parallel light, respectively. For the transmittance studies, two batches of PLA/m-CNF composite films with different thicknesses were fabricated. The thinner films were prepared by reducing the total amount of reactant to $1 \mathrm{~g}$ while maintaining their ratios. The morphologies of the PLA/m-CNF composite films were observed by SEM (SU3500, Hitachi, Tokyo, Japan) using Au-Pd sputter to increase the sample's surface conductivity. The samples were cut and attached to circular SEM plates $(25 \mathrm{~cm})$. For the surface morphology, magnification at $100 \times(500 \mu \mathrm{m}$ scale) was used to provide a wide area of the film's surface. The mechanical properties of the composite films were investigated with a universal testing machine (EZ Graph, Shimadzu, Kyoto, Japan) following the JIS K6251-8 standard. Each sample was cut into dumbbell shapes for at least five tests and dried in an oven at $80{ }^{\circ} \mathrm{C}$ before analysis to remove any remaining solvent and moisture. The crosshead speed was $50 \mathrm{~mm} / \mathrm{min}$ with a load cell of $100 \mathrm{~N}$. The crystallinities of PLA/m-CNF composite films were calculated using differential scanning calorimetry (DSC) (EXSTAR 6000 DSC6220, SEIKO, Tokyo, Japan) from crystallization temperature $\left(\mathrm{T}_{\mathrm{c}}\right)$ and melting temperature $\left(\mathrm{T}_{\mathrm{m}}\right)$ areas. The crystallinities of the PLA composite films were calculated using the theoretical heat of fusion $\left(\Delta \mathrm{H}_{\mathrm{f}}\right)\left(93.1 \mathrm{~J} \mathrm{~g}^{-1}\right)[28]$.

$$
\Delta \mathrm{H}_{\mathrm{m}}-\Delta \mathrm{H}_{\mathrm{c}}=\Delta \mathrm{H}^{\prime}
$$




$$
\begin{gathered}
\frac{\Delta \mathrm{H}^{\prime}}{\Delta \mathrm{H}_{\mathrm{f}}} \times 100=\mathrm{X}_{\mathrm{c}} \% \\
\Delta \mathrm{H}_{\mathrm{f}}=93.1 \mathrm{~J} / \mathrm{g}
\end{gathered}
$$

where $\Delta \mathrm{H}_{\mathrm{m}}$ and $\Delta \mathrm{H}_{\mathrm{c}}$ are the enthalpies given during melting and crystallization, respectively, and $X_{c} \%$ is the degree of crystallinity. The wettability of the films was investigated by WCA measurement using a Drop Master DM300 contact angle meter (Kyowa Interface Science, Tokyo, Japan) with FAMAS basic software. Each sample was dried in an oven at $80^{\circ} \mathrm{C}$ before analysis. The WCAs were measured for at least seven specimens, and the four values with the least deviation were used to calculate the average WCA.

\section{Results and Discussion}

\subsection{Surface Modification of CNF}

ATR-IR was performed to observe the acyl groups that substituted the hydroxyl groups of CNF after modification. The ATR-IR spectra of the m-CNFs are shown in Figure 2. New peaks appeared at around $1730 \mathrm{~cm}^{-1}$, corresponding to the $\mathrm{C}=\mathrm{O}$ stretching vibration modes of the carbonyl group between $\mathrm{CNF}$ and the anhydrides. This indicates that the acyl groups from the anhydrides were incorporated into the $\mathrm{CNF}$ to become $\mathrm{CNFa}$, $\mathrm{CNFp}$, and $\mathrm{CNFb}$. Moreover, the increases in the intensities of the carbonyl peaks with increasing reaction time showed that the acylation processes were proportional to the reaction time. The intensities of the $-\mathrm{OH}$ deformation of water peaks at around $1650 \mathrm{~cm}^{-1}$ were higher than the intensities of the $\mathrm{C}=\mathrm{O}$ stretching peaks for all of the $\mathrm{m}-\mathrm{CNFs}$ after 1-h acylation. This changed when the acylation time was increased to $2 \mathrm{~h}$, where the intensities of the carbonyl peaks were higher than those of the $-\mathrm{OH}$ deformation of water peaks. Furthermore, the intensities of the $\mathrm{C}=\mathrm{O}$ stretching peaks were higher for 4-h acylation compared with both 1- and 2-h acylation, indicating that the DS for 4-h acylation was the highest among the studied reaction times.

\subsection{Degree of Substitution}

EDX spectroscopy has a probe depth of 1-3 $\mu \mathrm{m}$, while the average diameter of the $\mathrm{CNF}$ was about $63 \pm 17 \mathrm{~nm}$. The average diameter was calculated based on the SEM image in Figure 3. EDX indicates the composition of each element, and therefore the DS values of the CNF and $\mathrm{m}-\mathrm{CNFs}$ can be calculated. The modification percentages of the m-CNFs with respect to the reaction time are shown on Figure 4. The modification percentages increased with increasing reaction time from 1 to $4 \mathrm{~h}$. The rates of acylation of CNFa and CNFp were almost the same and exponentially increased with the reaction time, while CNFp showed a slightly higher acylation rate. However, the acylation rate of $\mathrm{CNFb}$ showed that the acylation rate was significantly lower for longer alkyl-chain substituent. Longer alkyl-chain of the anhydride (BA) leads to a slower rate reaction due to steric effect and lower surface area compared to AA and PA [29]. After 1-h reaction, only $9.8 \%$ of CNFb was modified, which was the lowest among the m-CNFs. For $4 \mathrm{~h}$ reaction time, $24.5 \%$ of $\mathrm{CNFb}$ was modified, while $29.4 \%$ and $31.4 \%$ of CNFa and CNFp were modified, respectively. The EDX results further verified that the acyl groups were chemically bonded to the CNF and acylation increased with increasing reaction time. The DS values of the m-CNFs were calculated from the percentage modified values using Equation (S4), and the results are given in Table 1 and Table S1. 

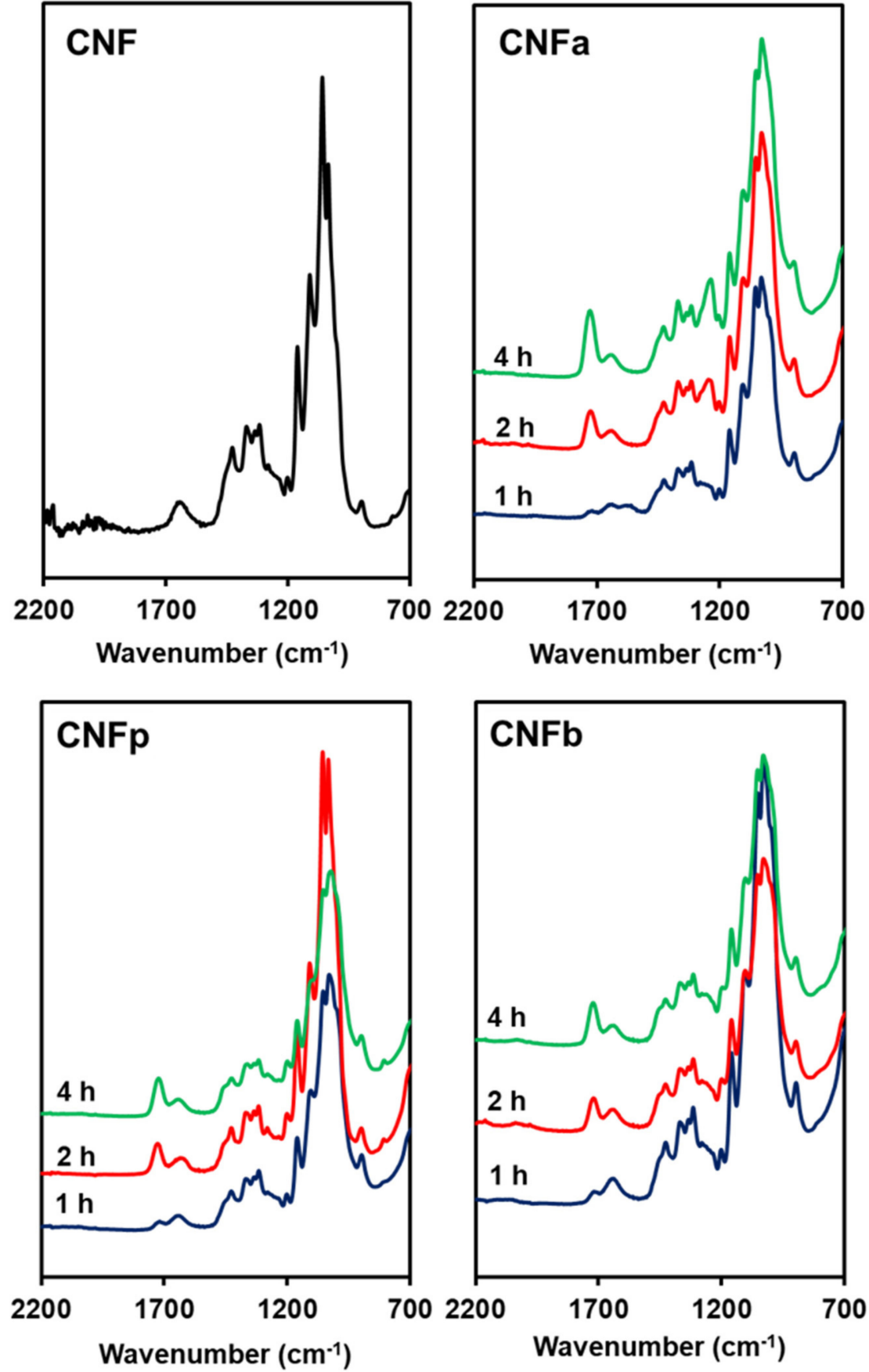

Figure 2. ATR-IR spectra of $\mathrm{CNF}, \mathrm{CNFa}, \mathrm{CNFp}$, and $\mathrm{CNFb}$.

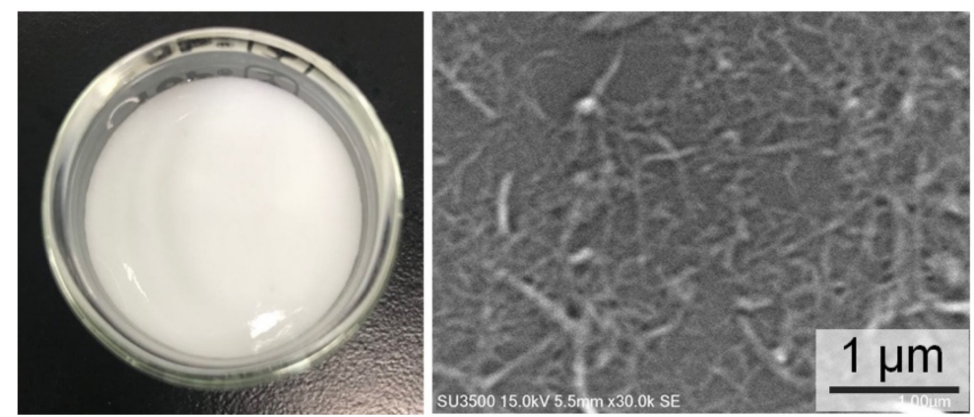

Figure 3. Suspension of CNF in water and its SEM image at $\times 30,000$ magnification. 


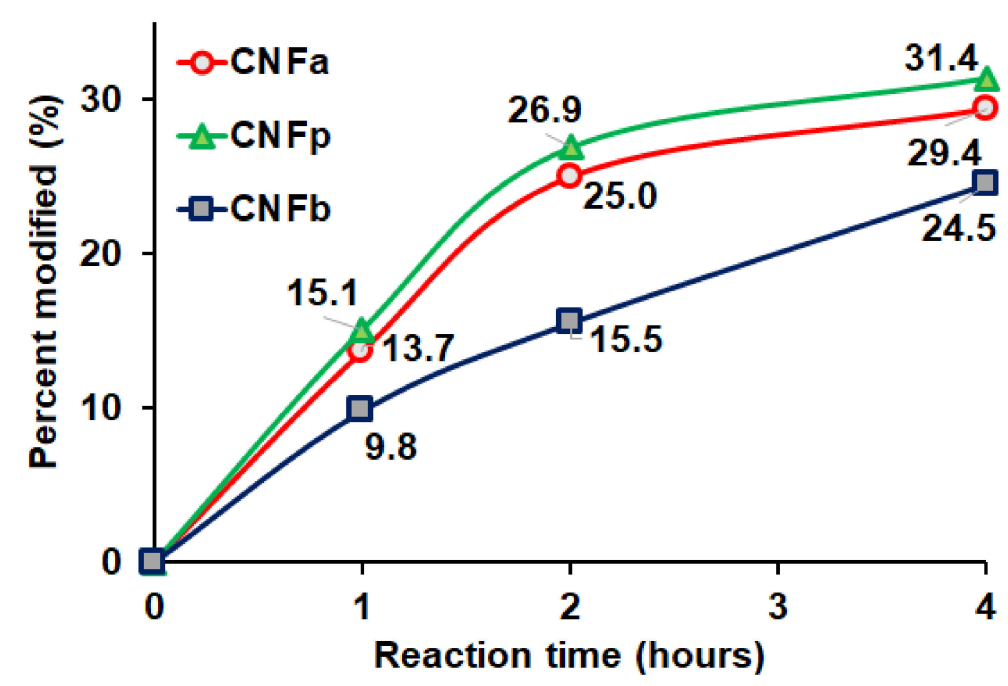

Figure 4. Percentage of $\mathrm{CNF}$ modified with respect to the reaction time for $\mathrm{CNFa}, \mathrm{CNFp}$, and $\mathrm{CNFb}$.

Table 1. Degrees of substitutions (DS) values of the modified CNFs (m-CNFs) based on the reaction time.

\begin{tabular}{cccc}
\hline \multirow{2}{*}{$\begin{array}{c}\text { Reaction Time } \\
\text { (Hours) }\end{array}$} & \multicolumn{3}{c}{ Degree of Substitution (DS) } \\
\cline { 2 - 4 } & CNFa & CNFp & CNFb \\
\hline 1 & 0.41 & 0.45 & 0.29 \\
2 & 0.75 & 0.81 & 0.47 \\
4 & 0.88 & 0.94 & 0.74 \\
\hline
\end{tabular}

\subsection{Optical Transmittance of the PLA/m-CNF Composite Films}

Photographs of the PLA/m-CNF composite films are shown in Figure 5. The patterns in the background can be clearly observed through the neat PLA film, demonstrating that the film possessed very good transparency (Figure 5a). The white agglomerates observed on the PLA/CNF composite film (Figure $5 b$ ) are because of its inhomogeneity with the PLA matrix. CNF tends to form agglomerates in the matrix because of their opposite hydrophilicity. Agglomerates of CNFa2, CNFp2, CNFa4, and CNFp4 in the PLA matrix were confirmed to be minimal because the background can still be clearly observed without any precipitation of the fillers. Even though the PLA/CNFb4 composite film (Figure $5 \mathrm{~h}$ ) exhibited high transparency because the background can be observed, the lower $\mathrm{DS}$ of $\mathrm{CNFb} 2$ (PLA/CNFb2 composite film) (Figure $5 \mathrm{~g}$ ) resulted in poor visibility of the background. This might be because the lower DS of CNFb2 (0.47) makes the unmodified $\mathrm{CNF}$ (in $\mathrm{CNFb} 2$ ) have a more significant effect on the PLA/m-CNF composite film, and hence reduces its miscibility with the PLA matrix. In addition, the surfaces of the films with the $\mathrm{CNFb}$ filler were rougher those with the CNFa and CNFp fillers regardless of their DS. As previously mentioned, the $\mathrm{m}-\mathrm{CNFs}$ (DS > 0.47) have high compatibility with PLA because of their similar hydrophobicity. For this reason, the PLA/CNFa, PLA/CNFp, and PLA/CNFb4 composite films showed better transparency than the PLA/CNF and $\mathrm{PLA} / \mathrm{CNFb} 2$ composite films. 

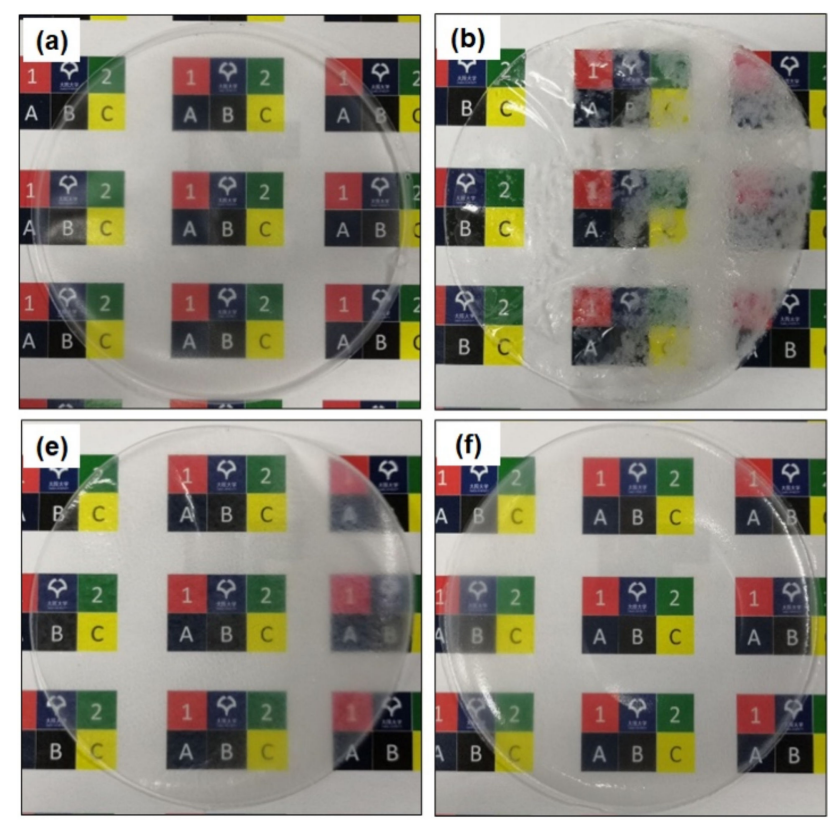
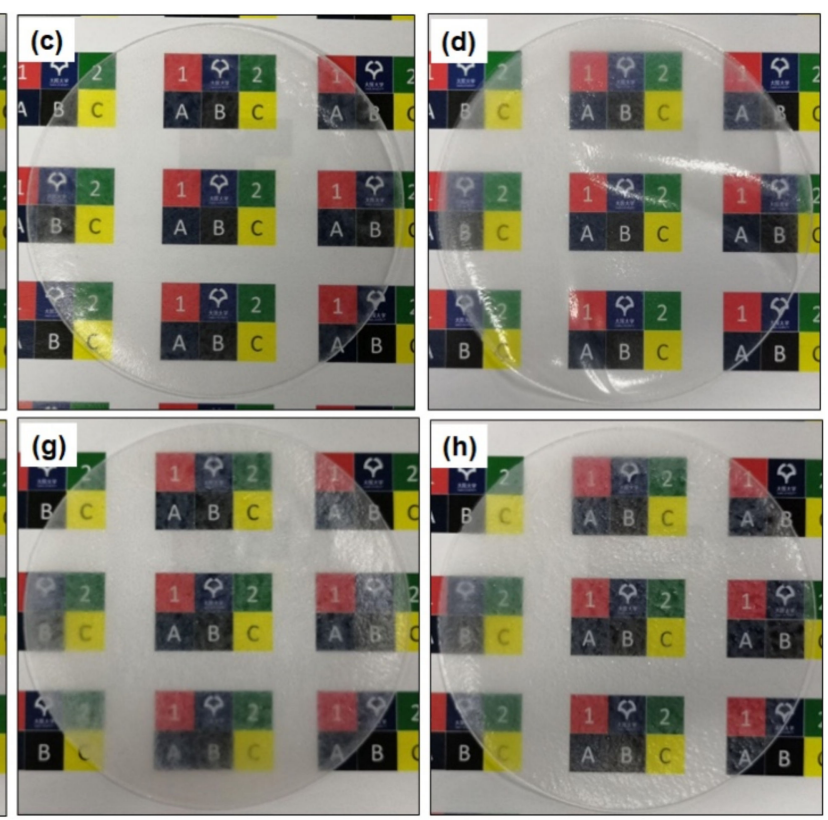

Figure 5. Photographs of the (a) neat PLA, (b) PLA/CNF, (c) PLA/CNFa2, (d) PLA/CNFa4, (e) PLA/CNFp2, (f) PLA/CNFp4, (g) PLA/CNFb2, and (h) PLA/CNFb4 composite films.

\subsection{Light Transmittance of the PLA/m-CNF Composite Films}

UV-vis spectroscopy was performed to compare the transparency of the PLA/m-CNF composite films by calculating the percentage of UV-vis transmittance. We compared two batches of PLA/m-CNF composite films with different thicknesses. The different thickness films were prepared by using different initial amounts of reactant ( 1 and $2 \mathrm{~g}$ ) during preparation of the films. The average transmittance values (Table 2) were used as relative values for comparison. Thicker films show lower transparency because the amount of light that passes through the films is reduced. All of the films prepared with a larger amount of reactant showed UV-vis transmittance below $42.2 \%$, including the neat PLA film. Furthermore, additional filler also decreases the transparency because the light is scattered/diffracted by the filler regardless of the thickness. Even though there was a very large difference between the UV-vis transmittance of the thick and thin films, both batches of PLA/m-CNF composite films showed the same pattern with respect to the filler. For $1 \mathrm{~g}$ of the reactant, the neat PLA film showed $89.3 \%$ transmittance, which was the highest among all of the films. The heterogeneous nature of the PLA/CNF composite film decreased the transmittance to $77.8 \%$. This is lower than that of the PLA film because of formation of agglomerates, which result in diffraction and scattering of light during UV-vis analysis. PLA with the $\mathrm{CNFb} 2$ filler ( $\mathrm{DS}=0.47)$ also showed lower transmittance $(77.9 \%)$ than the neat PLA film (89.3\%) owing to the low compatibility between the filler and PLA. The composite films of PLA with the other $\mathrm{m}-\mathrm{CNF}$ fillers showed better transmittance $(81.0-83.9 \%)$. This can be attributed to the better and more uniform dispersion of the relatively high DS $(\geq 0.74) \mathrm{m}-\mathrm{CNFs}$ in the PLA matrix compared with unmodified CNF or low DS m-CNFs. The transmittance spectra of the PLA $/ \mathrm{m}-\mathrm{CNF}$ composite films (1 g) in the visible wavelength region $(400-800 \mathrm{~nm})$ are shown in Figure 6, which were used to calculate the transparency values of the films. The transparency values were calculated based on the specific thickness of each film. All of the transparency values followed the same pattern as the UV-vis transmittance regardless of the amount of reactant used. 
Table 2. Average thickness, transmittance, and transparency values of the PLA/m-CNF composite films.

\begin{tabular}{cccccccc}
\hline \multirow{2}{*}{ Films } & \multirow{2}{*}{ DS of Fillers } & \multicolumn{2}{c}{ Thicknesses $(\boldsymbol{\mu m})$} & \multicolumn{2}{c}{ UV-Vis Transmittance (\%) } & \multicolumn{2}{c}{ Transparency Values } \\
\cline { 3 - 7 } & & $\mathbf{1}$ & $\mathbf{2} \mathbf{g}$ & $\mathbf{1} \mathbf{g}$ & $\mathbf{2} \mathbf{g}$ & $\mathbf{1} \mathbf{g}$ & $\mathbf{2} \mathbf{g}$ \\
\hline Neat PLA & - & 51 & 190 & 89.3 & 39.1 & 3.23 & 2.31 \\
PLA/CNF & - & 82 & 231 & 77.8 & 13.2 & 2.97 & 1.76 \\
PLA/CNFa2 & 0.75 & 65 & 238 & 81.0 & 26.8 & 3.08 & 2.05 \\
PLA/CNFp2 & 0.81 & 64 & 213 & 81.7 & 25.8 & 3.08 & 2.08 \\
PLA/CNFb2 & 0.47 & 81 & 232 & 77.9 & 25.3 & 2.95 & 2.04 \\
PLA/CNFa4 & 0.88 & 60 & 224 & 83.9 & 34.9 & 3.13 & 2.19 \\
PLA/CNFp4 & 0.94 & 57 & 177 & 81.2 & 42.2 & 3.14 & 2.38 \\
PLA/CNFb4 & 0.74 & 52 & 256 & 81.2 & 22.3 & 3.18 & 1.94 \\
\hline
\end{tabular}

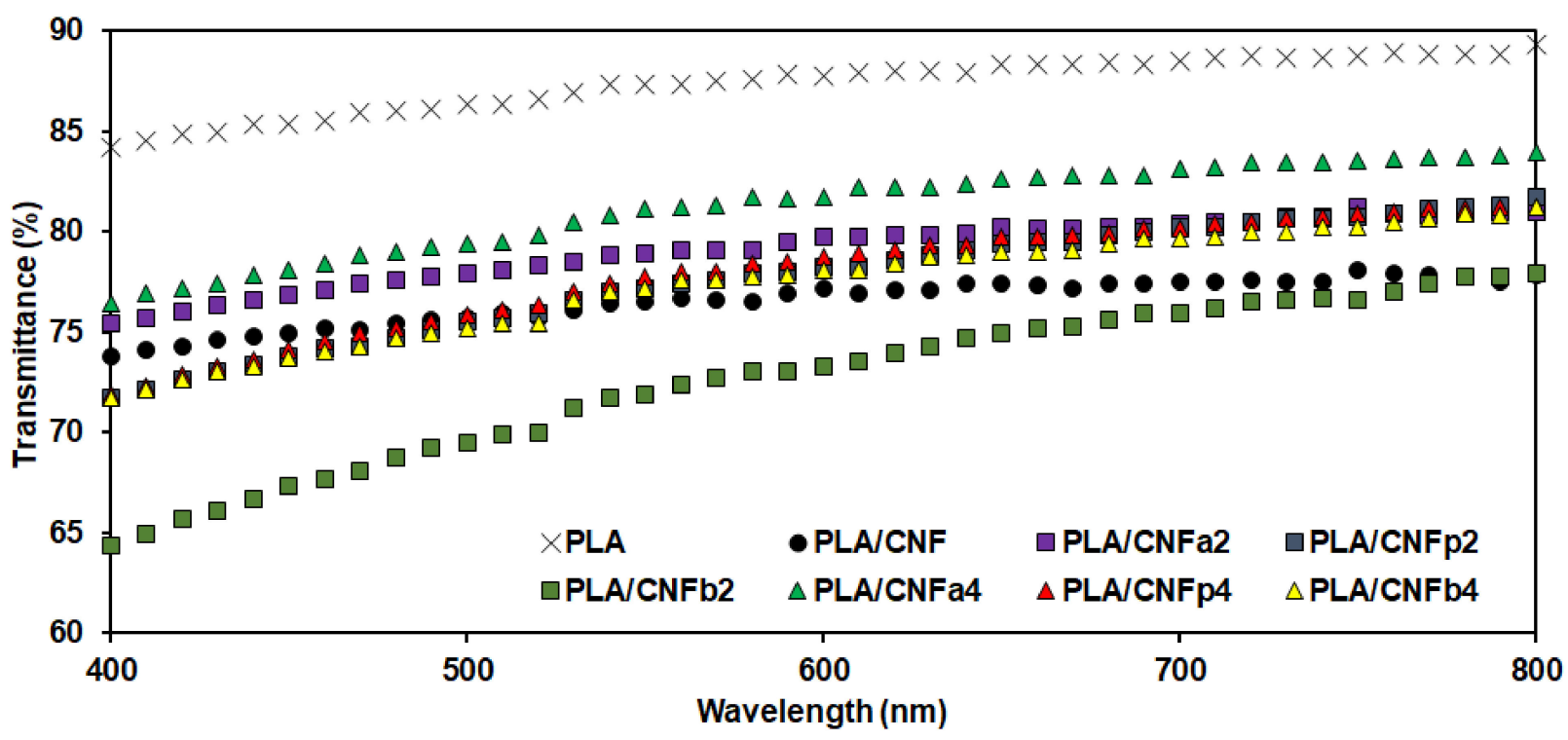

Figure 6. UV-vis transmittance of the PLA/m-CNF composite films (1 $\mathrm{g}$ of reactant).

\subsection{Haze Transmittance of the PLA/m-CNF Composite Films}

To further support the UV-vis transmittance, the haze values of the films were determined (Table 3). The haze values increased with addition of the fillers. The neat PLA film gave the lowest haze value of $6.8 \%$. The poor dispersions of $\mathrm{CNF}$ and $\mathrm{CNFb} 2$ showed haze values of $35.5 \%$ and $30.0 \%$, respectively. Adding the $\mathrm{m}-\mathrm{CNF}$ fillers changed the haze values to between $17.5 \%$ and $19.5 \%$ (except for $\mathrm{CNFb}$ ), which can be considered to be good for transparent films. The increase in the haze value from that of the neat PLA is because the fillers increased the amount of reflected and scattered light. The compatibility and dispersion of the higher DS m-CNF fillers in the PLA matrix are the key factors for achieving lower haze values compared with the PLA/CNF and PLA/CNFb2 composite films.

Table 3. Average thicknesses and haze values of the PLA/m-CNF composite films.

\begin{tabular}{cccc}
\hline Films & DS of Fillers & Thicknesses $(\boldsymbol{\mu m})$ & Haze Values $(\%)$ \\
\hline Neat PLA & - & 51 & 6.8 \\
PLA/CNF & - & 82 & 35.5 \\
PLA/CNFa2 & 0.75 & 65 & 19.5 \\
PLA/CNFp2 & 0.81 & 64 & 17.8 \\
PLA/CNFb2 & 0.47 & 81 & 30.0 \\
PLA/CNFa4 & 0.88 & 60 & 20.1 \\
PLA/CNFp4 & 0.94 & 57 & 17.5 \\
PLA/CNFb4 & 0.74 & 52 & 19.0 \\
\hline
\end{tabular}




\subsection{Morphologies of the PLA/m-CNF Composite Films}

The CNF filler resulted in different physical properties of the PLA matrix in comparison with the $\mathrm{m}-\mathrm{CNFs}$ fillers, especially on the surface, because of the difference in their compatibility. The PLA film (Figure 7a) showed a smooth surface because there was no filler involved. The PLA/CNF composite film had a rough surface (Figure $7 \mathrm{~b}$ ), and it was clear that the CNF filler caused agglomeration on/in the PLA matrix. Hence, the PLA/CNF composite film showed a lumpy surface with clear white agglomerates. In contrast, the PLA/m-CNF composite films with CNFa4 and CNFp4 as fillers (Figure 7d,f) exhibited flat and smooth surfaces because of their good compatibility with the PLA matrix. These results demonstrate that acylation changed the compatibility of the CNF with the PLA matrix for CNFa and CNFp. The relatively high DS m-CNF fillers were expected to have high compatibility with PLA owing to their hydrophobicity, while the hydrophilic $\mathrm{CNF}$ and relatively low DS m-CNFs were expected to have low compatibility with PLA. The SEM images showed that the scattered fillers on the surface were related to the DS of the $\mathrm{m}$-CNF fillers. As the DS of the $\mathrm{m}-\mathrm{CNF}$ decreased, the filler became more observable, as shown in Figure 7c,e. This indicates that the compatibility between the PLA matrix and $\mathrm{m}-\mathrm{CNF}$ filler is affected by the difference in the DS values. As discussed in Section 3.3, the surfaces of the PLA/CNFb composite films were both rough. The SEM images of the PLA/CNFb composite films (Figure $7 \mathrm{~g}, \mathrm{~h}$ ) are in agreement with those observations, because the PLA/CNFb composite films were rough. The DS of CNFb4 is 0.74 , which is almost the same as that of $\mathrm{CNFa} 2$ (DS = 0.75), so the difference in the lengths of the alkylchain substituents might be the cause of this opposite observation. It seems that the butyryl group substituted on the hydroxyl group of $\mathrm{CNF}$ tends to shield and localize $\mathrm{CNFb}$, and hence rough surfaces are observed for the PLA/CNFb composite films regardless of their DS. Morphology differences of the $\mathrm{m}-\mathrm{CNF}$ are observed in the SEM images (Figure S1). After modification with acid anhydrides, the structures of the m-CNFs changed, and higher $\mathrm{DS} \mathrm{m}$-CNFs produced smaller agglomerates. $\mathrm{CNFb} 4(\mathrm{DS}=0.74)$ formed relatively large agglomerates, which might lead to a rougher surface during PLA/CNFb composite film preparation. It appears that the higher number of the hydroxyl groups in CNFb4 (due to lower DS than CNFa4 and CNFp4) formed an opposite interaction between the unmodified and modified parts of $\mathrm{CNFb} 4$; hence, larger agglomerates were formed during dispersion in chloroform.
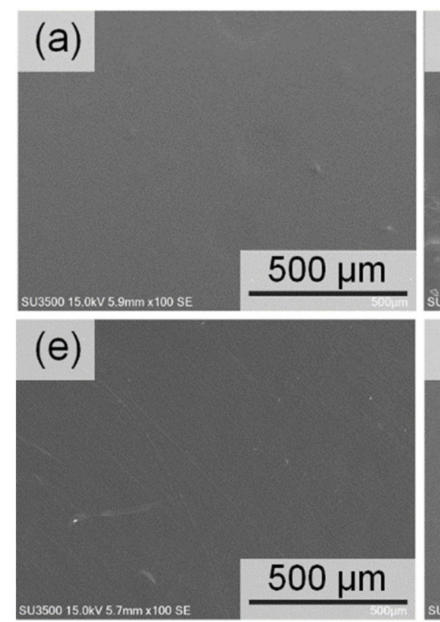


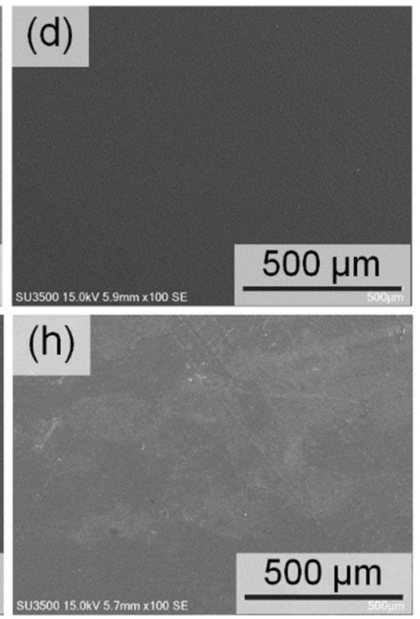

Figure 7. SEM images of the (a) neat PLA, (b) PLA/CNF, (c) PLA/CNFa2, (d) PLA/CNFa4, (e) PLA/CNFp2, (f) PLA/CNFp4, (g) PLA/CNFb2, and (h) PLA/CNFb4 composite films.

Other than surface morphologies, the cross-section of the films also was observed (Figure 8). It is interesting to note that neat PLA and PLA/CNF composite films display plastic-like deformation after cracked (Figure 8a,b). It was proven that CNF is not compati- 
ble with the PLA matrix. Meanwhile, Figure $8 \mathrm{c}-\mathrm{h}$ show that the m-CNFs are incorporated with the PLA matrix by forming stack-like morphologies.
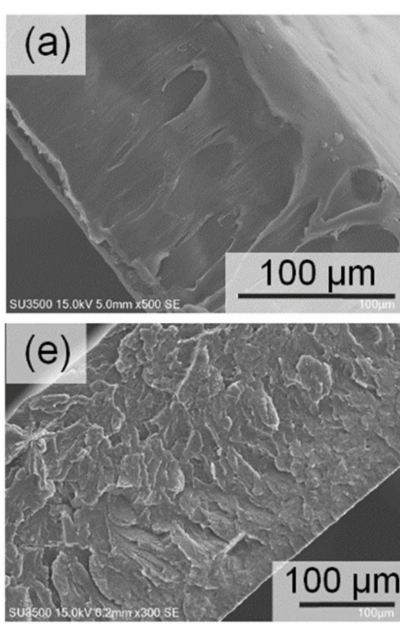
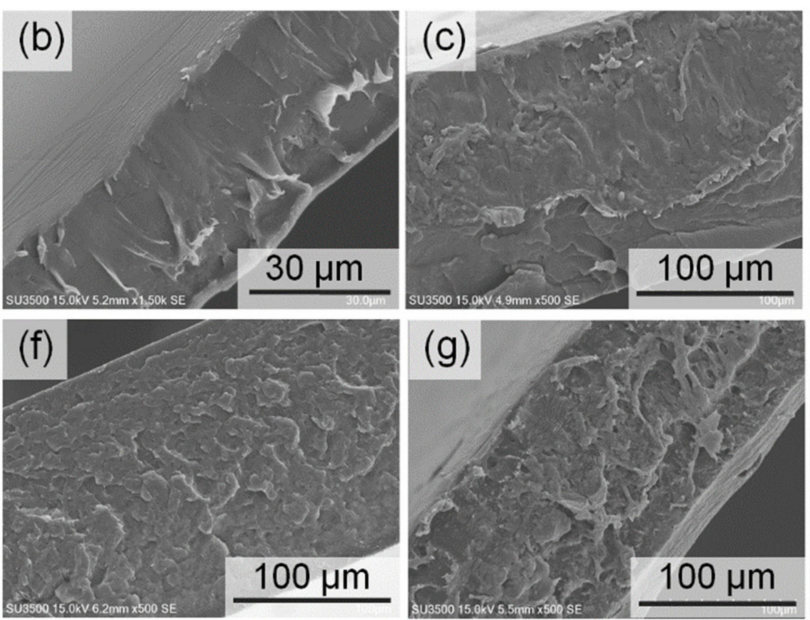
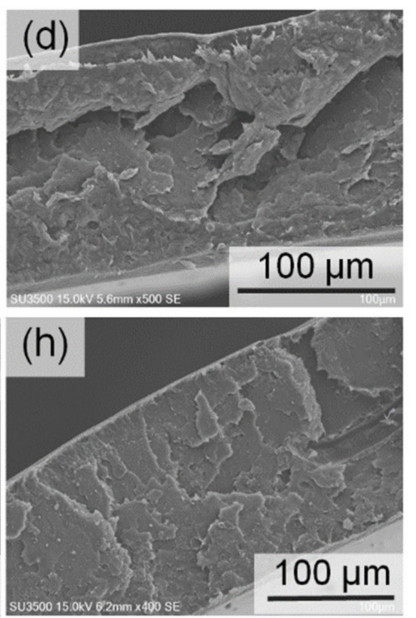

Figure 8. Cross-section images of the (a) neat PLA, (b) PLA/CNF, (c) PLA/CNFa2, (d) PLA/CNFa4, (e) PLA/CNFp2, (f) PLA/CNFp4, (g) PLA/CNFb2, and (h) PLA/CNFb4 composite films.

\subsection{Mechanical Properties of the PLA/m-CNF Composite Films}

The mechanical properties of the films are given in Table 4. The neat PLA film showed a tensile strength of $46.1 \pm 4.5 \mathrm{MPa}$, and inclusion of CNF in the PLA matrix decreased the tensile strength $(23.9 \pm 5.8 \mathrm{MPa})$. The decrease in the tensile strength of the PLA/CNF composite film was because of the inhomogeneity between the PLA matrix and CNF filler. The DS of the m-CNF played an important role in determining the tensile strength of the PLA/m-CNF composite film. Regardless of the filler species, all of the films with fillers of $\mathrm{DS}<0.76$ (CNFa2, $\mathrm{CNFb} 2$, and $\mathrm{CNFb} 4)$ exhibited lower tensile strength than the neat PLA film. Conversely, the fillers with DS $>0.80$ showed higher tensile strength, with the PLA/CNFp4 composite film showing the highest tensile strength of $53.0 \pm 2.4$ $\mathrm{MPa}$. Both the CNFa4 (DS $=0.88$ ) and CNFp2 (DS =0.81) fillers also improved the tensile strength of the PLA/m-CNF composite films compared with the neat PLA film. Therefore, the tensile strength of the PLA film can be improved by addition of a m-CNF with DS > 0.80. In general, composites prepared by the solution casting method have lower tensile strength than those prepared by the melt blend method [30]. This also occurred for our products because the tensile strength of PLA was lower than that of pure commercialized or industrial PLA. The neat PLA film showed a Young's modulus of $1.24 \pm 0.28 \mathrm{GPa}$. The differences among the Young's modulus values of the PLA/m-CNF composite films showed the same pattern as the tensile strength, except for the CNFa fillers. Even though the PLA matrix with the CNFa2 filler showed lower tensile strength than the neat PLA film (by 11\%), its Young's modulus was about 14\% higher. In contrast, the PLA/CNFa4 composite showed higher tensile strength than the neat PLA film, but its Young's modulus was lower $(1.07 \pm 0.10 \mathrm{GPa})$. Regarding the strain to failure, there were no significant differences among the strain percentages of the PLA/m-CNF composite films (2.4-3.9\% with standard deviations of 0.1-1.4). The areas under the strain-stress curves (Figure S2) were calculated to predict the tensile toughness values of the films. Although the $\mathrm{PLA} / \mathrm{m}-\mathrm{CNF}$ composite films showed good tensile strength and modulus, their toughness was relatively poor, the same as general PLA [31]. 
Table 4. Average tensile strength, Young's modulus, tensile strain, and toughness of the PLA/m-CNF composite films.

\begin{tabular}{ccccc}
\hline Composite Films & Tensile Stress $\mathbf{( M P a )}$ & Young's Modulus (GPa) & Tensile Strain (\%) & Toughness $\left(\mathbf{J} / \mathbf{m m}^{\mathbf{3}}\right)$ \\
\hline Neat PLA & $46.1 \pm 4.5$ & $1.24 \pm 0.28$ & $3.6 \pm 0.5$ & $1.54 \pm 0.21$ \\
PLA/CNF & $23.9 \pm 5.8$ & $1.00 \pm 0.39$ & $2.5 \pm 0.4$ & $0.25 \pm 0.08$ \\
PLA/CNFa2 & $41.1 \pm 0.2$ & $1.41 \pm 0.57$ & $3.3 \pm 1.4$ & $0.49 \pm 0.03$ \\
PLA/CNFp2 & $49.4 \pm 3.0$ & $1.75 \pm 0.08$ & $3.0 \pm 0.4$ & $0.80 \pm 0.14$ \\
PLA/CNFb2 & $32.5 \pm 1.4$ & $1.23 \pm 0.17$ & $2.4 \pm 0.3$ & $0.90 \pm 0.59$ \\
PLA/CNFa4 & $50.5 \pm 2.1$ & $1.07 \pm 0.10$ & $3.9 \pm 0.5$ & $1.65 \pm 0.01$ \\
PLA/CNFp4 & $53.0 \pm 2.4$ & $1.74 \pm 0.09$ & $3.2 \pm 0.1$ & $1.31 \pm 0.21$ \\
PLA/CNFb4 & $36.3 \pm 0.1$ & $1.08 \pm 0.21$ & $0.81 \pm 0.18$ \\
\hline
\end{tabular}

\subsection{Crystallinity of the PLA/m-CNF Composite Films}

Both transparency and mechanical properties of PLA can be affected by the crystallinity of the samples [32]. Calculated from DSC thermograms (Figure S3), the $X_{c}$ of $\mathrm{PLA} / \mathrm{m}-\mathrm{CNF}$ composite films is obtained and reported in Table $5 . \mathrm{X}_{\mathrm{c}}$ of PLA $/ \mathrm{m}-\mathrm{CNF}$ composite films was lower compared to neat PLA (39.6\%) due to the formation of aggregates, which decreased the number of nucleating sites [33]. PLA/m-CNF composite films showed that no significant variation was observed regardless of the m-CNFs' substituent groups, which vary from $30.7 \%$ to $33.1 \%$. Meanwhile, PLA/CNF composite film showed the most amorphous structure at $\mathrm{X}_{\mathrm{c}}$ of $16.2 \%$ due to the incompatibility between CNF filler and PLA matrix, hence forming more aggregates than m-CNFs fillers. Therefore, PLA/CNF composite films exhibited a lower amount of nucleating sites.

Table 5. The degree of crystallinity for PLA/m-CNF composite films.

\begin{tabular}{cc}
\hline Films & Degree of Crystallinity, $\mathbf{X}_{\mathbf{c}} \mathbf{( \% )}$ \\
\hline Neat PLA & 39.6 \\
PLA/CNF & 16.2 \\
PLA/CNFa2 & 32.4 \\
PLA/CNFp2 & 28.2 \\
PLA/CNFb2 & 28.1 \\
PLA/CNFa4 & 30.7 \\
PLA/CNFp4 & 33.1 \\
PLA/CNFb4 & 28.6 \\
\hline
\end{tabular}

\subsection{Wettability of the PLA/m-CNF Composite Films}

The WCAs of the neat PLA and PLA/m-CNF composite films are shown in Figure 9. The neat PLA film showed an average WCA of $83.9^{\circ}$, whereas that of the PLA/CNF composite film was $81.7^{\circ}$. This shows that the PLA/CNF filler had higher wettability owing to the hydrophilicity of CNF. All of the PLA/m-CNF composite films showed higher WCAs than the neat PLA and PLA/CNF films regardless of the filler and DS. This is attributed to a change from hydrophilic $\mathrm{CNF}$ to hydrophobic $\mathrm{CNFa}, \mathrm{CNFp}$, and $\mathrm{CNFb}$. Substitution of the acyl groups for the hydroxyl groups of CNF is the main reason for the hydrophobicity. From the discussion in Section 3.1, the number of hydrogen bonds of CNF decreases when the hydroxyl groups are substituted by hydrophobic acyl groups. Therefore, the wettability of the PLA film also decreases by adding m-CNF fillers compared with unmodified CNF. Based on the results in Figure 9, the length of the alkyl-chain substituent had a significant effect on the wettability of the PLA/m-CNF composite film. Comparing the fillers with the same acylation time, PLA/CNFb showed the highest WCA values for both reaction times $\left(87.3^{\circ}\right.$ and $88.5^{\circ}$ for the $\mathrm{CNFb} 2$ and $\mathrm{CNFb} 4$ fillers, respectively). This indicates that the substituted propionyl group of $\mathrm{CNFb}$ increased the hydrophobicity of the $\mathrm{PLA} / \mathrm{m}-\mathrm{CNF}$ composite film even though the DS was lower than those of both CNFa and CNFp. Comparison between CNFa and CNFp also showed that the CNFa fillers had lower hydrophobicity (WCAs of $85.3^{\circ}$ and $86.0^{\circ}$ for $\mathrm{CNFa} 2$ and $\mathrm{CNFa} 4$, respectively) owing to their shorter alkyl-chain substituents. 


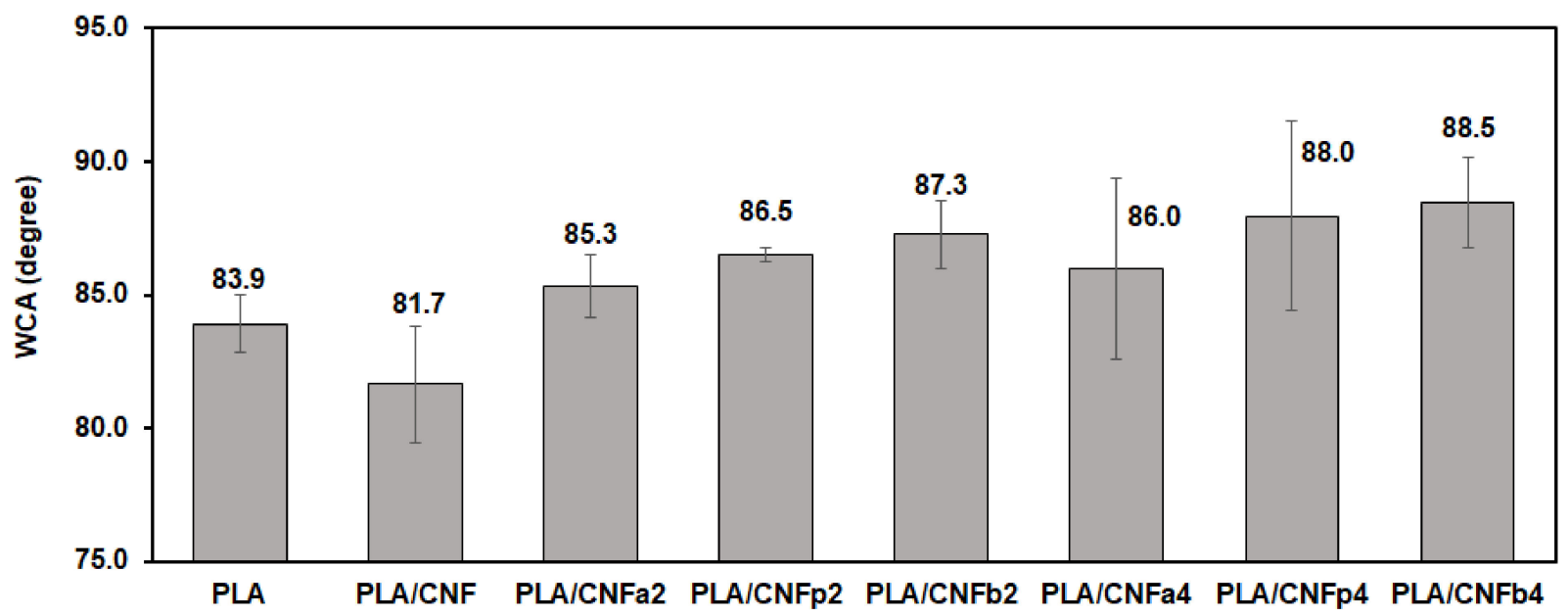

Figure 9. Wettability of the PLA composite films.

\section{Conclusions}

In this study, CNF was acylated using acid anhydrides with short alkyl-chain substituents ( $\mathrm{C} 1$ to $\mathrm{C} 3$ ), followed by fabrication of PLA/m-CNF composite films. CNFp showed the highest rate of acylation among the $\mathrm{m}-\mathrm{CNFs}$, and reaction for $4 \mathrm{~h}$ produced $31.4 \%$ CNFp, which was the highest among all of the studied parameters. The introduction of $\mathrm{CNF}$ as a filler decreased the transparency, mechanical, and wettability properties compared with the neat PLA film. Conversely, the compatibility of the m-CNFs with the PLA matrix mostly improved. By comparison of the films, the effects of different $\mathrm{m}-\mathrm{CNF}$ s on PLA were investigated. The PLA/CNFp4 composite films showed the best transparency and mechanical properties, which was mainly because CNFp4 had the highest DS. Therefore, the DS of the filler is the key factor to improve the properties of PLA/m-CNF composite films. Meanwhile, the low DS CNFb2 filler ( $\mathrm{DS}=0.47$ ) showed inhomogeneity of the matrix-filler interaction because the unmodified CNF part of $\mathrm{CNFb} 2 \mathrm{had}$ a more significant effect on the composite. Thus, the transparency and mechanical properties of the PLA/CNFb2 composite film were lower than those of the other PLA/m-CNF films. However, according to the WCA, the wettability of the PLA/CNFb2 composite film improved. Based on these observations, it was concluded that longer alkyl-chain substituent $(\mathrm{CNFb}$ filler) had a greater influence than the DS of the filler on the wettability properties of the PLA/m-CNF composite films.

Supplementary Materials: The following are available online at https:/ / www.mdpi.com/2079-499 1/11/3/753/s1, Table S1: Details of the mass concentrations obtained from EDX analysis, Figure S1: SEM images of (a) CNF, (b) CNFa4 (DS = 0.88), (c) CNFp4 (DS = 0.94), and (d) CNFb4 (DS = 0.74) at $\times 3000$ magnification, Figure S2: Stress-strain curves of PLA/m-CNF composite films, Figure S3: DSC thermograms of PLA/m-CNF composite films.

Author Contributions: N.J.: investigation, methodology, visualization, writing-original draft. Y.-I.H.: conceptualization, funding acquisition, writing-review and editing. T.-A.A.: funding acquisition, writing-review and editing. H.U.: conceptualization, funding acquisition, supervision, writing-review and editing. All authors have read and agreed to the published version of the manuscript.

Funding: This work was funded by JSPS KAKENHI Grants number 19H02778, 20H02797, and 20K15343, and the JST-Mirai Program Grant number JPMJMI18E3.

Data Availability Statement: The data presented in this study are available within the article or Supplementary Materials.

Acknowledgments: N.J. would like to thank the Japanese Government through the Ministry of Education, Culture, Sports, Science and Technology of Japan (MEXT) for a scholarship. We thank 
Tim Cooper, from Edanz Group (https: / / en-author-services.edanzgroup.com/, 16 February 2021) for editing a draft of this manuscript.

Conflicts of Interest: The authors declare no conflict of interest.

\section{References}

1. Grande, D.; Ramier, J.; Versace, D.L.; Renard, E.; Langlois, V. Design of functionalized biodegradable PHA-based electrospun scaffolds meant for tissue engineering applications. New Biotechnol. 2017, 37, 129-137. [CrossRef]

2. Rasal, R.M.; Janorkar, A.V.; Hirt, D.E. Poly(lactic acid) modifications. Prog. Polym. Sci. 2010, 35, 338-356. [CrossRef]

3. Arrieta, M.P.; Samper, M.D.; Aldas, M.; López, J. On the Use of PLA-PHB Blends for Sustainable Food Packaging Applications. Materials 2017, 10, 1008. [CrossRef] [PubMed]

4. Bor, Y.; Alin, J.; Hakkarainen, M. Electrospray Ionization-Mass Spectrometry Analysis Reveals Migration of Cyclic Lactide Oligomers from Polylactide Packaging in Contact with Ethanolic Food Simulant. Packag. Technol. Sci. 2012, 25, 427-433. [CrossRef]

5. Murariu, M.; Dubois, P. PLA composites: From production to properties. Adv. Drug Deliv. Rev. 2016, 107, 17-46. [CrossRef] [PubMed]

6. Arrieta, M.P.; Peponi, L. Polyurethane based on PLA and PCL incorporated with catechin: Structural, thermal and mechanical characterization. Eur. Polym. J. 2017, 89, 174-184. [CrossRef]

7. Alemdar, A.; Sain, M. Isolation and characterization of nanofibers from agricultural residues-Wheat straw and soy hulls. Bioresour. Technol. 2008, 99, 1664-1671. [CrossRef]

8. Iwamoto, S.; Nakagaito, A.N.; Yano, H. Nano-fibrillation of pulp fibers for the processing of transparent nanocomposites. Appl. Phys. A 2007, 89, 461-466. [CrossRef]

9. Saito, T.; Isogai, A. Introduction of aldehyde groups on surfaces of native cellulose fibers by TEMPO-mediated oxidation. Colloids Surf. A Physicochem. Eng. Asp. 2006, 289, 219-225. [CrossRef]

10. Zhang, Y.; Li, H.; Li, X.; Gibril, M.E.; Han, K.; Yu, M. Green chemical preparation of cellulose/high performance elastomer blend fibers by melt-spinning method. J. Polym. Res. 2013, 20, 1-9. [CrossRef]

11. Jamaluddin, N.; Kanno, T.; Asoh, T.-A.; Uyama, H. Surface modification of cellulose nanofiber using acid anhydride for poly(lactic acid) reinforcement. Mater. Today Commun. 2019, 21, 100587. [CrossRef]

12. Shalwan, A.; Yousif, B. In State of Art: Mechanical and tribological behaviour of polymeric composites based on natural fibres. Mater. Des. 2013, 48, 14-24. [CrossRef]

13. Fujisawa, S.; Saito, T.; Kimura, S.; Iwata, T.; Isogai, A. Surface Engineering of Ultrafine Cellulose Nanofibrils toward Polymer Nanocomposite Materials. Biomacromolecules 2013, 14, 1541-1546. [CrossRef]

14. Lee, K.-Y.; Blaker, J.J.; Bismarck, A. Surface functionalisation of bacterial cellulose as the route to produce green polylactide nanocomposites with improved properties. Compos. Sci. Technol. 2009, 69, 2724-2733. [CrossRef]

15. Bae, J.H.; Kim, S.H. Alkylation of mixed micro- and nanocellulose to improve dispersion in polylactide. Polym. Int. 2015, 64, 821-827. [CrossRef]

16. Qu, P.; Zhou, Y.; Zhang, X.; Yao, S.; Zhang, L. Surface modification of cellulose nanofibrils for poly(lactic acid) composite application. J. Appl. Polym. Sci. 2012, 125, 3084-3091. [CrossRef]

17. Quero, F.; Eichhorn, S.J.; Nogi, M.; Yano, H.; Lee, K.-Y.; Bismarck, A. Interfaces in Cross-Linked and Grafted Bacterial Cellulose/Poly(Lactic Acid) Resin Composites. J. Polym. Environ. 2012, 20, 916-925. [CrossRef]

18. Pei, A.; Zhou, Q.; Berglund, L.A. Functionalized cellulose nanocrystals as biobased nucleation agents in poly(l-lactide) (PLLA) Crystallization and mechanical property effects. Compos. Sci. Technol. 2010, 70, 815-821. [CrossRef]

19. Saito, T.; Kimura, S.; Nishiyama, Y.; Isogai, A. Cellulose Nanofibers Prepared by TEMPO-Mediated Oxidation of Native Cellulose. Biomacromolecules 2007, 8, 2485-2491. [CrossRef]

20. Cui, X.; Honda, T.; Asoh, T.-A.; Uyama, H. Cellulose modified by citric acid reinforced polypropylene resin as fillers. Carbohydr. Polym. 2020, 230, 115662. [CrossRef] [PubMed]

21. Cui, X.; Ozaki, A.; Asoh, T.-A.; Uyama, H. Cellulose modified by citric acid reinforced Poly(lactic acid) resin as fillers. Polym. Degrad. Stab. 2020, 175, 109118. [CrossRef]

22. Dixon, J.; Andrews, P.; Butler, L.G. Hydrophobic esters of cellulose: Properties and applications in biochemical technology. Biotechnol. Bioeng. 1979, 21, 2113-2123. [CrossRef]

23. Vaca-Garcia, C.; Gozzelino, G.; Glasser, W.G.; Borredon, M.E. Dynamic mechanical thermal analysis transitions of partially and fully substituted cellulose fatty esters. J. Polym. Sci. Part B Polym. Phys. 2002, 41, 281-288. [CrossRef]

24. Crépy, L.; Chaveriat, L.; Banoub, J.; Martin, P.; Joly, N. Synthesis of Cellulose Fatty Esters as Plastics-Influence of the Degree of Substitution and the Fatty Chain Length on Mechanical Properties. ChemSusChem 2009, 2, 165-170. [CrossRef] [PubMed]

25. Duchatel-Crépy, L.; Joly, N.; Martin, P.; Marin, A.; Tahon, J.-F.; Lefebvre, J.-M.; Gaucher, V. Substitution degree and fatty chain length influence on structure and properties of fatty acid cellulose esters. Carbohydr. Polym. 2020, 234, 115912. [CrossRef] [PubMed]

26. Teramoto, Y. Functional Thermoplastic Materials from Derivatives of Cellulose and Related Structural Polysaccharides. Molecules 2015, 20, 5487-5527. [CrossRef] 
27. Yu, W.; Han, N.; Qian, Y.; Zhang, X.; Li, W. Effects of Fatty Acid Anhydride on the Structure and Thermal Properties of Cellulose-g-Polyoxyethylene (2) Hexadecyl Ether. Polymer 2018, 10, 498. [CrossRef]

28. Fischer, E.W.; Sterzel, H.J.; Wegner, G. Investigation of the structure of solution grown crystals of lactide copolymers by means of chemical reactions. Kolloid Z. Z. Polym. 1973, 251, 980-990. [CrossRef]

29. DeTar, D.F. Effects of alkyl groups on rates of SN2 reactions. J. Org. Chem. 1980, 45, 5174-5176. [CrossRef]

30. Khurma, J.R.; Rohindra, D.R.; Devi, R. Miscibility study of solution cast blends of poly(lactic acid) and poly(vinyl butyral). S. Pac. J. Nat. Appl. Sci. 2005, 23, 22-25. [CrossRef]

31. Farah, S.; Anderson, D.G.; Langer, R. Physical and mechanical properties of PLA, and their functions in widespread applicationsA comprehensive review. Adv. Drug Deliv. Rev. 2016, 107, 367-392. [CrossRef] [PubMed]

32. Kun, E.; Marossy, K. Effect of Crystallinity on PLA's Microbiological Behaviour. Mater. Sci. Forum 2013, 752, 241-247. [CrossRef]

33. Perić, M.; Putz, R.; Paulik, C. Influence of nanofibrillated cellulose on the mechanical and thermal properties of poly(lactic acid). Eur. Polym. J. 2019, 114, 426-433. [CrossRef] 